\title{
COMMENTARY
}

\section{Optimizing management of patients with microhematuria: Gold down the drain?}

\author{
Christopher J.D. Wallis, MD, PhD, FRCSC ' Zachary Klaassen, MD, MSc ${ }^{2,3}$
}

'Department of Urology, Vanderbilt University Medical Center, Nashville, TN, United States; ${ }^{2}$ Division of Urology, Medical College of Georgia at Augusta University, Augusta, GA, United States; ${ }^{3}$ Georgia Cancer Center, Augusta, GA, United States

Cite as: Can Urol Assoc J 2019;13(12):412-3. http://dx.doi.org/10.5489/cuai.6315

See related article on page 406

M icroscopic hematuria $(\mathrm{MH})$ is a common reason for urological consultation. The evaluation and management of $\mathrm{MH}$ presents a clinical dilemma, as evidenced by differing guidelines across jurisdictions in Canada, Europe, and the U.S. Due to both its relatively high incidence and involved workup, $\mathrm{MH}$ may account for proportionately high health expenditures given the relatively low incidence of underlying malignancy in these patients. However, the potential to miss an opportunity for early diagnosis of bladder cancer is a risk few urologists would embrace. The key, therefore, lies in optimizing selection of patients for $\mathrm{MH}$ investigation and optimizing the investigation undertaken in order to maximize benefit while reducing morbidity and cost. To this end, Assmus and colleagues undertook an audit of the practices of two Edmonton urologists. ${ }^{1}$ Two main conclusions arise from this work.

First, despite relative unanimity among guidelines, there is some difficulty with the operationalization of the definition of $\mathrm{MH}$. The Canadian Urological Association (CUA) guideline clearly defines $\mathrm{MH}$ as greater than two red blood cells per high-powered field (RBC/HPF) on two separate urinalyses, without provoking factors. ${ }^{2}$ Assmus et al point out that the laboratory reporting in their jurisdiction actually precluded accurate assessment according to this definition (instead using 1-5 RBC/HPF). Notably, when assessed further, the majority of patients (59\%) with 1-5 RBC/HPF actually had $1-2 \mathrm{RBC} / \mathrm{HPF}$ and, thus, did not meet criteria for $\mathrm{MH}(25 \%$ of all patients evaluated!). However, the authors do not comment on how many of these patients had repeated testing demonstrating persistent $\mathrm{MH}$. Presumably a not insignificant portion would fail to demonstrate $\mathrm{MH}$ on a second sample. Together, this highlights that significant efficiencies could streamline the number of patients undergoing $\mathrm{MH}$ workup simply by adhering to existing guidelines.
Second, the authors should be congratulated for their fastidious adherence to the CUA guideline. However, in doing so, they demonstrate the low incidence of underlying pathology in these patients, suggesting an opportunity to further triage patients prior to investigation. Not a single patient in their cohort had a malignant cytology result. Cystoscopy was normal in $86 \%$ of patients, with incidental findings accounting for $91 \%$ of the remainder. Three malignant bladder tumors and two more inflammatory lesions were identified cystoscopically, of which one would have been found on imaging. Similarly, upper tract imaging was normal in the vast majority and of those with demonstrated abnormality, findings were incidental and not requiring intervention in the majority. Of the non-malignant "significant" pathology, the vast majority would be expected to present symptomatically before harm befell the patient. Thus, it may be worthwhile to consider whether existing guidelines could become more stringent, reducing the number of negative evaluations with the incumbent healthcare cost and patient burden.

In the U.S., use of computed tomography urography, rather than ultrasound, for upper tract evaluation of patients with $\mathrm{MH}$ is both widespread and guideline-advocated. ${ }^{3}$ Especially given the low yield of upper tract findings demonstrated by Assmus and colleagues, as well as many others, the risks of such an approach, compared to ultrasound, likely outweigh any diagnostic benefit. ${ }^{4}$ Other work has similarly demonstrated that the combined use of cystoscopy and ultrasound in the initial evaluation of $\mathrm{MH}$, as advocated in CUA guideline, is the most cost-effective approach. ${ }^{5}$ Thus, for patients warranting investigation, the approach laid out in the CUA guideline appears to maximize outcomes both for the patient and the healthcare system.

In 2003, Dr. Malmstrom advocated abandoning testing for $\mathrm{MH}$ on account of the poor predictive value for underlying malignancy and poor sensitivity and specificity in distinguishing urological and non-urological conditions of the abdomen and pelvis. ${ }^{6}$ While it is important to not step this far, Assmus and colleagues have certainly demonstrated that there remains significant room for optimization in the man- 
agement of these patients. They demonstrate cost savings of $\$ 745000$ annually simply from changing the reporting of urinalysis results. This is likely to underestimate the true benefit, given that it only accounts for costs from the payer perspective. Given the low underlying rate of significant pathology in these patients, more stringent patient selection may further reduce the costs and burdens of investigation. It seems that the true gold flowing down the drain in this circumstance is from healthcare coffers.

Competing interests: The authors report no competing personal or financial interests related to this work.

\section{References}

1. Assmus MA, Beyer D, Hanks J, et al. Quality and cost assessment of Canadian Urological Association microscopic hematuria guidelines in clinical practice: Turning urine into gold. Can Urol Assoc J2019;13:40611. http://dx.doi.org/10.5489/cuaj.5809

2. Wollin T, Laroche B, Psooy K. Canadian guidelines for the management of asymptomatic microscopic hematuria in adults. Can Urol Assoc J 2009;3:77-80. https://doi.org/10.5489/cuaj.1029

3. Davis R, Jones IS, Barocas DA, et al. Diagnosis, evaluation, and followup of asymptomatic microhematuria (AMH) in adults: AUA guideline. J Urol 2012;188:2473-81. https://doi.org/10.1016/i. juro.2012.09.078

4. Yecies T, Bandari J, Fam M, et al. Risk of radiation from computerized tomography urography in the evaluation of asymptomatic microscopic hematuria. J Urol 2018;200:967-72. https://doi.org/10.1016/i. juro.2018.05.118

5. Halpern JA, Chughtai B, Ghomrawi H. Cost-effectiveness of common diagnostic approaches for evaluation of asymptomatic microscopic hematuria. JAMA Int Med 2017;177:800-7. https://doi.org/10.1001/ jamainternmed.2017.0739

6. Malmstrom PU. Time to abandon testing for microscopic haematuria in adults? BMJ 2003;326:813-5. https://doi.org/10.1136/bmi.326.7393.813

Correspondence: Dr. Christopher J.D. Wallis, Department of Urology, Vanderbilt University Medical Center, Nashville, TN, United States; wallis.cjd@gmail.com

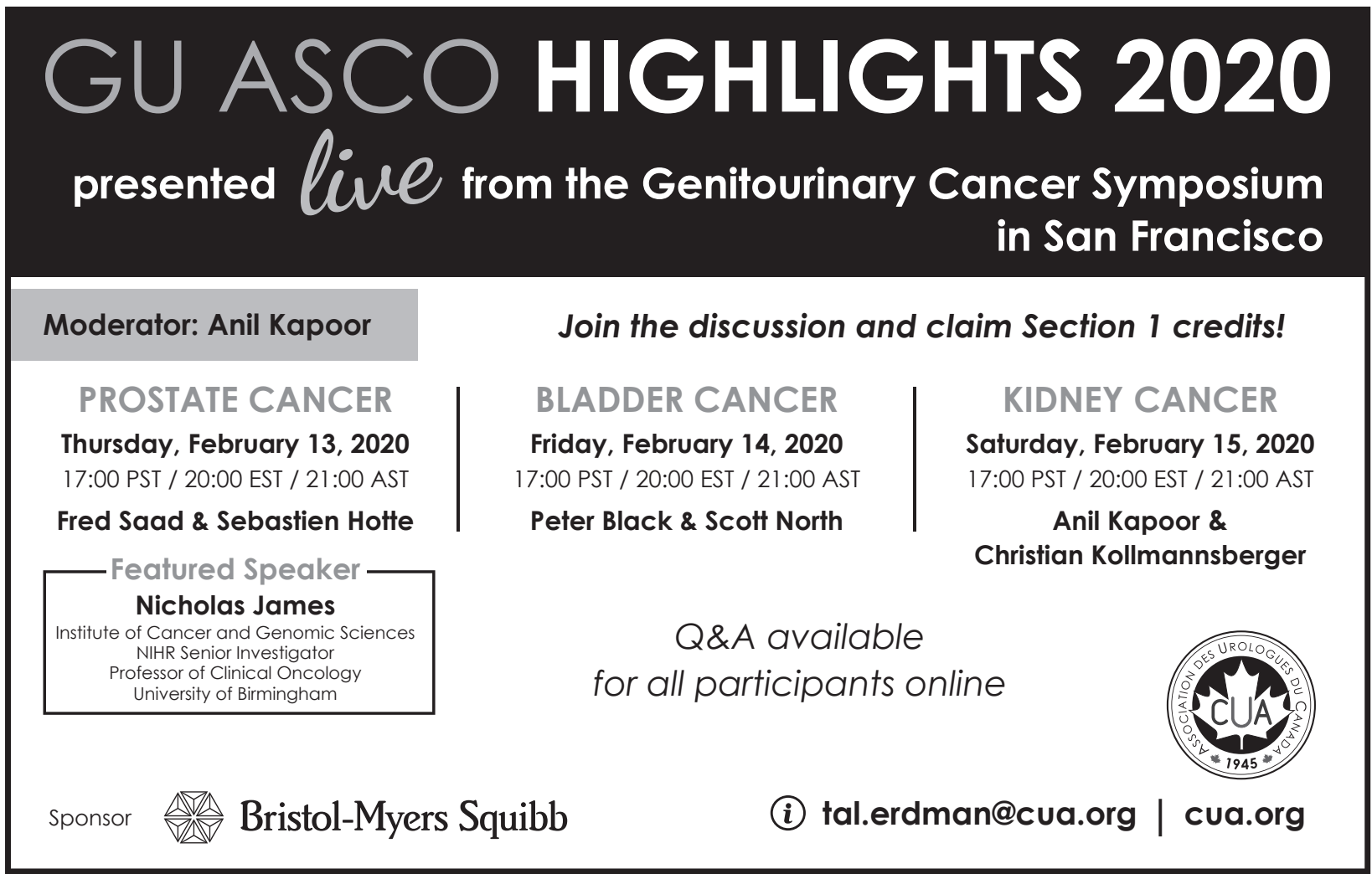

